Case Report

\title{
Pre-surgical Orthopedic (PSO) Pre-maxilla Appliance for Children with Cleft Lip and Palate: A Case Series
}

\author{
Tania Saskianti, Rizki Amalia, Stephanie Clara Limbri, Ardianti Maartrina Dewi, Udijanto \\ Tedjosasongko \\ Department of Pediatric Dentistry, \\ Faculty of Dental Medicine, Universitas Airlangga, \\ Surabaya - Indonesia
}

\begin{abstract}
Background: Cleft lip and palate is one of most common craniofacial anomalies. It is a congenital defect and can be resulted from genetic mutation, environment and multifactor causes. The problems of cleft lip and palate on baby complicate as poor feeding ability can lead to growth and development disturbances. Purpose: This article described the use of PSO in a 4-day old male neonate with unilateral cleft lip and palate and a 3-year old male child with soft and hard palate cleft, both with chief complaint of feeding difficulty. Case Management: Custom tray was used to deliver the alginate impression. The appliance was both fabricated using self-cured acrylic and with / without lip strapping. Discussion: Pre-surgical orthopedic appliances are most frequently provided by the pediatric dentist on cleft palate teams at most hospital-based programs. Conclusion: The PSO appliance could approximate the segments of the cleft maxilla by allowing spontaneous growth of palatal shelves prior to the reconstructive and definitive surgery.
\end{abstract}

Keywords: Pre-surgical orthopaedics, cleft lip and palate, impression, children

Correspondence: Tania Saskianti, Department of Pediatric Dentistry, Faculty of Dental Medicine, Universitas Airlangga, Jl. Prof. Dr. Moestopo 47 Surabaya 60132 - Indonesia, Phone: +62315030255. Email: tanias@fkg.unair.ac.id

\section{INTRODUCTION}

Cleft lip and palate (CLP) occurred predominantly in males, with male: female predilection is $2: 1^{1}$. Poor feeding ability in CLP neonates have a bad impact in weight, led to growth and development disturbances. Numerous dental anomalies and malocclusions occur with cleft lip or palate. Therefore, pediatric dentist should discuss with patients and the parents about the dental problems associated with clefting ${ }^{2}$. Pre-surgical Orthopaedic (PSO) as initial treatment of CLP with new technique can greatly improve upon facial aesthetics and minimize the extent of the surgery and surgical procedures ${ }^{3}$. The objective of the article was to report the use of PSO in a 4-day old male neonate with unilateral cleft lip and palate and a 3year old male child with soft and hard palate cleft, both with chief complaint of feeding difficulty.

\section{CASES}

Case 1: A 4-day old male neonate with CLP came to Pediatric Dental Clinic, Universitas Airlangga Dental Hospital with chief complaint of feeding difficulty. The infant was born by caesarean at 33 weeks and weigh 2700 grams. Case 2: A 3year-old male child came to Pediatric Dental Clinic, Universitas Airlangga Dental Hospital with chief complaint of a reopened palatum. Recently, the child had palate surgical repair about 7 months ago (Figure 1A, 1B, 1C). 
Volume 1 Issue 1 2018; 5-9
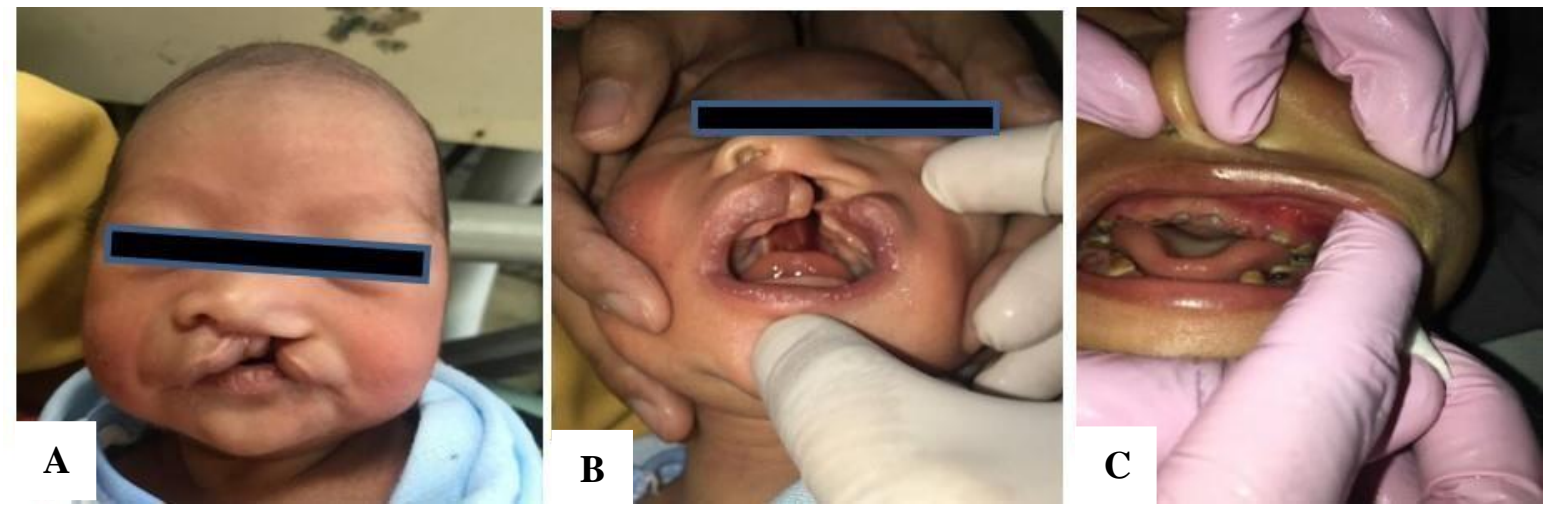

Figure 1 A, B, C Extra-oral and Intraoral photographs

In both cases, there was no contributory medical history of mother during pregnancy. Moreover, no history of CLP from parents and relatives. Extra oral and intra oral examination showed unilateral complete CLP (Veau's classification, class III). A resin acrylic PSO was chosen and alginate impression was used.

During the impression procedure, the patient was conscious without any anaesthesia/ premedication. The patient was held face down and crying to enable a continuously potent airway. The infant was not allowed to drink 2 hours prior to impression procedure, to ovoid and vomit.
A custom tray was used in delivering the alginate. After fully set, the impression was removed and inspected whether the anatomy was fully recorded and ensured there was no alginate left in the mouth. The cast was made with dental plaster and then lubricated with petroleum jelly. The defect in palate was blocked with wax to avoid undercuts. The tray was fabricated using self-cured acrylic with "sprinkle" method where small portions powder and liquid of clear acrylic were incrementally added to cast $^{2}$. In addition, dental floss was added for easier removal and to avoid accidental swallowing/ choking (Figure 2).

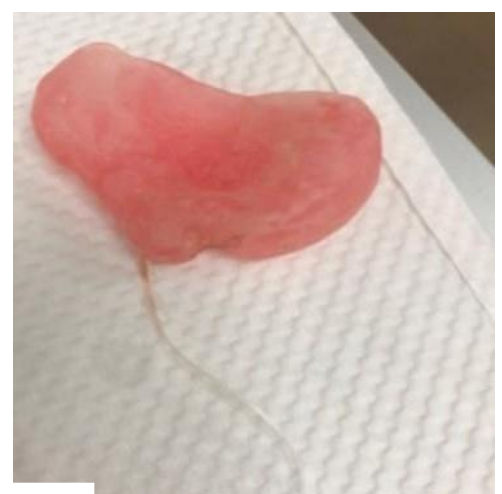

A

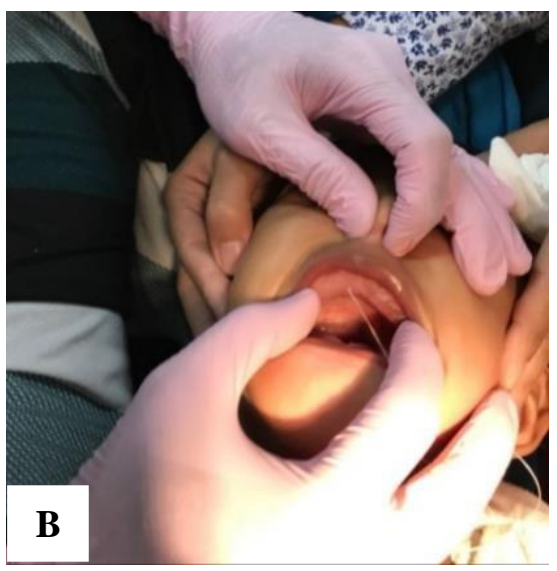

Figure 2. The PSO was ready for insertion 
All the peripheral borders were polished properly to avoid mucosal irritation. The appliance was combined with lip strap using external tapes strapped to the cheeks. The parents were instructed on: use of the appliance, its placement, removal and daily cleaning. If there was any discomfort, pain or ill-fitting appliance, the parents were suggested for a visit.

The first case, one month follow up showed appliance was well-fitted and there was no discomfort pain. A new dental floss was attached to the appliance. The infant's weight has increased 500 grams because, and 1100 grams on next the 2nd months of adequate milk. From examination, the appliance was loose-fitted due to maxilla growth. Therefore, a new PSO with naso-alveolar molding (NAM) was made. An acrylic custom tray was made with a wax sheet of approximate size and shape and, then adapted intraorally. As soon as the wax was placed in the oral cavity with index and middle finger, the baby started suckling. During this period, the wax was molded to the palate extending to muco-buccal fold areas. The wax mould was removed from mouth with minimum of distortion. Then, the dental plaster cast was prepared to fabricate an acrylic custom impression tray ${ }^{1}$. The impression and fabrication procedure was obtained similarly as mentioned in first visit. PSO combined with NAM was fabricated with resin acrylic. The nasal stent is made of 0.6 $\mathrm{mm}$ - gauge round stainless steel wire. It extended forward and then curves backward (a swan neck form), entering 3 to $4 \mathrm{~mm}$ past the nostril aperture. Then, hard acrylic component is shaped into a bilobed form that resembles a kidney. The upper lobe entered the nose and gently lifted the dome until a moderate amount of tissue blanching is evident. The lower lobe of the nasal stent lifts the nostril apex and defines the top of the columella. The appliance was secured extra-orally to the cheeks and bilaterally by surgical tapes.

The second case, three-months follow up showed the appliance was ill-fitted so grinding and polishing was done to readjust the appliance. There were lots of teeth remnants and decayed teeth. The child's weight had increased around 1000 grams and the patient was referred to Oral Maxillo facial and Surgery Department for palatopasty.

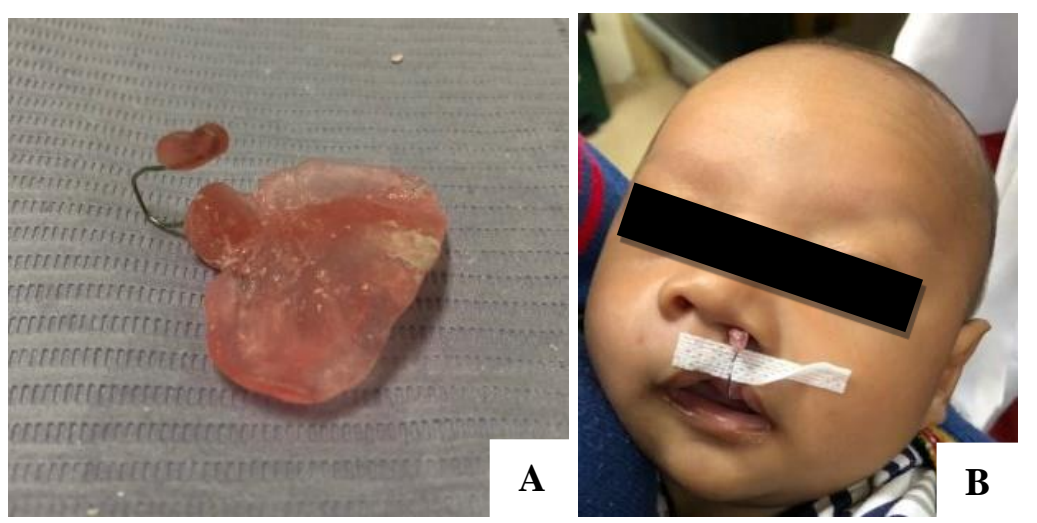

Figure 3. Two month follow up: new PSO combined with NAM 


\section{DISCUSSION}

Neonates with a cleft palate have difficulty in eating, which may lead to failure to thrive. The oronasal communications results in demised ability to create negative pressure that is necessary for sucking. Nasal regurgitation, excessive air intake and choking are other frequent complications ${ }^{4}$.

Pediatric dentistry plays a critical role in creating a proper plan of care for oral health and overall nutrition. Pre-surgical orthopedic appliances are most frequently provided by the pediatric dentist on cleft palate teams at most hospital-based programs ${ }^{4}$.The main objective of PSO is to approximate the palatal shelves closely to each other prior to surgical repair of lip and palate. Besides, PSO is aimed to facilitate intra-oral feeding, improve maxillary growth and the projection of the nasal tip, reduce nasal deformity, facilitate primary lip, nasal, and alveolar surgeries, also retract and reposition the premaxilla more posteriorly in patients with bilateral cleft ${ }^{5}$. Surgical lip repair may be accomplished at 10 weeks of age. Early repair of the palate may have a negative effect on the growth and development of maxilla due to the resulting scartissue ${ }^{4}$.

The ideal time for the PSO treatment is from birth to 5 months of age, which will have the advantage of faster correction in less time ${ }^{6}$. Therefore, case 1 presented a more easily adapted appliance while applied to the 4-day old infant's mouth. In the opposite, case 2 showed a higher difficulty for a 3-year old child wearing and adapting to the appliance, resulted in poor prognosis. Unilateral CLP can be treated using passive appliance and active appliance with lip strapping ${ }^{5}$.In case 1, PSO appliance with lip strap was chosen because of significant cleft in palatum extending to lip with protruding premaxilla. While, case 2 introduced PSO appliance without additional features because defect only occurred in palatum. When there is only little distortion, passive appliance can be applied in order to keep tongue away and facilitate lateral palatal shelf growth, while active appliance and lip strapping was used when there is significant distortion on the cleft area. Elastic forces of lip strap will exert are tracting, backward pressure against the protruding premaxilla and careful use of forces on the cleft segments will improve their positions and allow definitive lip skin and muscle repair ${ }^{5}$.

Furthermore, in case 1, PSO with additional NAM was considerably made in order to reduce the severity of the initial cleft deformity. In the short term, the benefit of NAM is achieving well aligned tissues before primary lip and nose repair, which enables the surgeon to achieve a better and more predictable outcome with less scar tissue formation. In the long term, the change in nasal shape is stable with less scar tissue and better lip and nasal form. This improvement reduces the number of surgical revisions for excessive scar tissue, oronasal fistulas, nasal and labial deformities. Tapes that actively bring the lip segments together are used in conjunction with the molding plate and nasal stent. Taping the lips together helps to make the inclined columella upright along the midsagittal plane. With the alveolar segments in a better position and increased bone bridges across the cleft, the adult teeth have a better chance of erupting in a good position with adequate periodontal support ${ }^{3}$.

In case 2, lots of teeth remnant and decayed teeth were found because of high sugar diet that causes a major concern as it tremendously raises the risk of caries. The parents should be trained to and educate their children about healthy eating habits ${ }^{7}$.

The impression procedure is an important process in the fabrication of the appliance. Patient positioning, tray and impression materials selections need to be considered thoroughly. The infant was held with his face towards the floor in order to prevent aspiration in case of vomiting and also asphyxiation due to airway obstruction .

Some of impression materials used are alginate, elastomeric (rubber base) and low fusing impression compound. The alginate was used in the case because its ability to record the details in the presence of saliva, inexpensive, easy to manipulate and comfortable to the patient ${ }^{9}$. From our experience, putty-type polyvinylsiloxane yields less accurate reproduction of surface details due to its high viscosity property compared to alginate. Although it can minimize complications such as aspiration/swallowing during the impression procedure ${ }^{8}$. 
There are some materials available for appliance's fabrication, which are low density polyethylene, acrylic resins and soft silicone rubbers ${ }^{9}$. In these cases, PSO was made from acrylic resin because it has good strength, fast and easy to manipulate, low cost and comfortable to wear. Besides, it can function as a rigid platform, towards which the child can press the nipple and feed; and also directs the tongue in to the correct position. Proper tongue position will be useful for developing normal speech and restoring well-functioned mastication $^{5,6}$. The case revealed that The PSO appliance could approximate the segments of the cleft maxilla by allowing spontaneous growth of palatal shelves prior to the reconstructive and definitive surgery.

\section{REFERENCES}

1. Bansal R, Pathak AK, Bhatia B, Gupta S, Gautam KK. Rehabilitation of a One-dayOld Neonate with Cleft Lip and Palate using Palatal Obturator: A Case Report. International Journal of Clinical Pediatric Dentistry 2012;5(2):145-147.

2. Mc Donald RE, Dean JA, Avery DR. Dentistry for the Child and Adolescent. $10^{\text {th }}$ edition 2011, Mosby Elsevier: Missouri p. 616-24.
3. Grayson BH, Maull D. Nasoalveolar Molding for Infants Born with Clefts of the Lip, Alveolus, and Palate. Seminars in Plastic Surgery 2005; 19(4): 294-301.

4. Hussein AM, Watted N, Hussien E, Watted A. A Feeding Appliance for A Newborn Baby with Cleft Palate. International Journal Dental and Medical Sciences Research 2017; 1(6): 05-09.

5. Alzain I, Batwa W, Cash A, Murshid Z.A. Pre-surgicalCleft Lip and PalateOrthopedics: an Overview. Clinical Cosmetic and Investigational Dentistry 2017; 9: 53-59.

6. Ellore VPK, Ramagoni NK, Taranatha M, Nara A, Gunjalli G, Bhat AD. Pre-surgical Orthopedic Pre-Maxillary Alignment in Bilateral Cleft Lip and Palate Patient. ContempClin Dent 2012; 3(3): 359-362.

7. Doichinova L, Bakardijev P, Peneva M. Assessment of Food Habits in Children aged 6-12 Years and the Risk of Caries. Biotecnology \& Biotechnological Equipment 2015; 29(1): 200- 204.

8. Chandna P, Adlakha VK, Singh N.Feeding Obturator Appliance for an Infant with Cleft Lip and Palate. Journal of Indian Society of Pedodontics and Preventive Dentistry 2011; 29(1): 71-73.

9. Ravichandra KS, Vijayaprasad KE1, Vasa AAK, Suzan S. A New Technique of Impression Making for anObturator in Cleft Lip and Palate Patient. Journal of Indian Society of Pedodontics and Preventive Dentistry 2010; 28(4): 311-314. 